Christoph A. Rass

\title{
Die Anwerbeabkommen der Bundesrepublik Deutschland mit Griechenland und Spanien im Kontext eines europäischen Migrationssystems
}

Zu Beginn der 1960er Jahre gewann die Arbeitswanderung von der europäischen Peripherie in die führenden Volkswirtschaften Westeuropas beträchtliche Dynamik. Seit dem Ende des Zweiten Weltkriegs hatten vor allem Migranten aus Italien die Nachfrage der Arbeitsmärkte zunächst Frankreichs und Belgiens, dann Luxemburgs und schließlich auch Deutschlands gedeckt. Nun aber stand das europäische System der Arbeitswanderung vor seiner ersten großen Erweiterung in der Nachkriegszeit. Immer mehr Staaten warben Arbeiterinnen und Arbeiter in Italien an oder förderten deren selbstständige Migration. Die ständig und zusehends schneller steigende Nachfrage nach Arbeitskraft in jedem einzelnen dieser Länder, in denen die Hochkonjunktur der „Trente Glorieuses“ Tritt fasste, begann das Migrationspotential Italiens, dieser lange Zeit unangefochten wichtigsten Quelle für Arbeitswanderer, zu erschöpfen. ${ }^{1}$

Die bevorstehende Diversifizierung der Migrationsverhältnisse auf dem europäischen Arbeitsmarkt deutete nicht allein das Einsetzen noch weitgehend unregulierter, teils klandestiner Wanderungen von Arbeitsuchenden aus Spanien, Portugal, Griechenland oder der Türkei in eine wachsende Zahl von Zielländern in Westeuropa an. Sie manifestierte sich auch auf der Ebene der bilateralen Wanderungsverträge, die ein Element der staatlich regulierten Anwerbung von Arbeitskräften waren. Schon Ende der 1950er Jahre begannen vielfach Verhandlungen auf zwischenstaatlicher Ebene, die ab Anfang der 1960er Jahre zu einer sich schnell auftürmenden Welle von Neuabschlüssen führten. Waren zwischen 1946 und 1959 insgesamt 15 auf temporäre Arbeitsmigration gerichtete Wanderungsabkommen in Westeuropa zustande gekommen, so folgten zwischen 1960 und 1973/74 - als die westeuropäischen Industriestaaten die Anwerbung einstellten - 45 Abkommen unter Berücksichtigung von Folgeverträgen. Der Abschluss von 37 (82 Prozent) dieser Verträge entfiel auf das Jahrzehnt zwischen 1960 und 1969. An seinem Ende sahen sich die wichtigsten Ab- und Zuwanderungsländer des europäischen Migrationssystems durch das dichteste Netz bilateraler Anwerbeabkommen miteinander verwoben, das zwischen den westeuropäischen Industriestaaten und ihrer ökonomischen Peripherie je existiert hatte. ${ }^{2}$

Am Anfang dieser außerordentlichen Verdichtung der durch bilaterale Wanderungsverträge formalisierten Migrationsbeziehungen standen im Jahr 1960 die Abkommen der Bundesrepublik Deutschland mit Spanien und Griechenland. ${ }^{3}$ Zwar hatten beide Staaten bereits zuvor Vereinbarungen ähnlicher Art abgeschlossen - Griechenland mit Frankreich 1954 und Belgien 1957, Spanien 1956 mit Belgien -, deren Einfluss auf die Wanderungs-

\footnotetext{
${ }^{1}$ Klaus J. Bade, Europa in Bewegung. Migration vom späten 18. Jahrhundert bis zur Gegenwart, München 2002, S.314f.

${ }^{2}$ Christoph A. Rass, Institutionalisierungsprozesse auf einem internationalen Arbeitsmarkt. Bilaterale Wanderungsverträge in Europa zwischen 1919 und 1974, Paderborn 2010, S.466f. Als Teil dieses Systems der Arbeitsmigration werden im Folgenden als Zuwanderungsländer Belgien, Deutschland, Frankreich, Luxemburg, die Niederlande, Osterreich, Schweden sowie die Schweiz aufgefasst. Als Abwanderungsländer gehörten ihm Algerien, Griechenland, Italien, Jugoslawien, Marokko, Polen (bis 1939/45), Portugal, Spanien, die Türkei sowie Tunesien an.

${ }^{3}$ Auf eine direkte Quellenangabe für alle zitierten Wanderungsabkommen wird in diesem Beitrag aus Platzgründen verzichtet. Ein Verzeichnis findet sich bei Rass, Institutionalisierungsprozesse, S.497507.
} 
ströme hatte sich jedoch als marginal erwiesen. ${ }^{4}$ Tatsächlich markiert erst der deutschspanische Wanderungsvertrag gemeinsam mit seinem deutsch-griechischen Pendant in vielfacher Hinsicht eine Zäsur in der Entwicklung des durch bilaterale Verträge konstituierten europäischen Arbeitsmarktes und seiner Wanderungsdynamik.

Deutschland erlangte durch diese Diversifizierung seiner Migrationsbeziehungen Zugang zu zwei wichtigen neuen Quellen für die im „Wirtschaftswunder“ so dringend benötigten Arbeitskräfte. Zugleich erschlossen sich Spanien und Griechenland einen privilegierten und gesicherten Migrationskanal zu einem sehr aufnahmefähigen Arbeitsmarkt, und damit eine Gelegenheit, über Arbeitsmigration sozialen Druck abzuleiten und durch die Rücküberweisung von Lohnersparnissen gewissermaßen in Devisenzuflüsse umzuwandeln. Daneben gewann das Netz von Wanderungsabkommen, welches das Migrationssystem zugleich abbildete und formte, eine Komplexität, in der Konkurrenzeffekte um Arbeitsplätze oder Arbeitskräfte eine gänzlich unerwartete Wirkung entfalten konnten. Dies wiederum ging Hand in Hand mit der sich ihrem Höhepunkt nähernden Bedeutung des institutionellen Rückgrats des Systems selbst - der Wanderungsverträge.

Aus diesen Perspektiven betrachtet der vorliegende Beitrag die beiden Anwerbeabkommen, die Deutschland mit Griechenland bzw. Spanien im Jahr 1960 abschloss, und beantwortet die Fragen: Was bedeuteten die Verträge für die Bundesrepublik? Wie wirkten sie sich auf die Wanderungsverhältnisse zwischen den drei beteiligten Staaten, wie auf die Position Griechenlands und Spaniens im weiteren Kontext der europäischen Arbeitswanderung bis zum Anwerbestopp aus? Welche Rolle spielten die Verträge von 1960 für das gesamte Vertragsnetzwerk? Was bedeuteten sie schließlich für die institutionelle Ausformung des bilateralen Wanderungsvertrages? Das Ziel ist eine exemplarische differenzierende Analyse, die eine nationalstaatliche mit der internationalen Ebene verbindet, dabei weder das Migrationssystem noch das ihm zugrunde liegende Vertragsnetz aus dem Blick verliert und eine für die Arbeitsmigration in Europa nach dem Zweiten Weltkrieg wichtige Phase diskutiert.

\section{Das europäische Migrationssystem vor und nach 1945}

Arbeitswanderung in Europa vollzog sich zwischen dem Ende des Ersten Weltkriegs und dem „Ölpreisschock“ Anfang der 1970er Jahre weder in voneinander isolierten bilateralen Migrationsbeziehungen noch auf einem multilateralen Arbeitsmarkt gleichberechtigter Partner, sondern in einem Migrationssystem, das aus einer Vielzahl interdependenter Verbindungen zwischen den Arbeitsmärkten einer stetig wachsenden Staatengruppe bestand. Die eingebundenen Arbeitsmärkte erfuhren dadurch eine grenzüberschreitende Erweiterung. Hinsichtlich der bestimmenden Wanderungsströme hatte die Entwicklung dieses Systems bereits im späten 19.Jahrhundert ihren Ausgang genommen, als die moderne grenzüberschreitende Mobilität von Arbeitskräften zum vorherrschenden Moment in der europäischen Arbeitswanderung wurde. Darunter wird eine durch den sektoralen Wandel bedingte, zunächst temporäre Arbeitswanderung größeren Umfangs aus relativ unterentwickelten Regionen in sich industrialisierende Staaten verstanden. ${ }^{5}$

Das Wanderungssystem besaß zwei Ebenen. Zum einen war es gekennzeichnet durch die grenzüberschreitenden Mobilitäts- bzw. Migrationsmuster, zum anderen durch ein sich

\footnotetext{
${ }^{4}$ Ebenda, S. $159 \mathrm{f}$.

${ }^{5}$ Christoph A. Rass, Bilaterale Wanderungsverträge und die Entwicklung eines internationalen Arbeitsmarktes in Europa 1919-1974, in: Ute Frevert/Jochen Oltmer (Hrsg.), Europäische Migrationsregime. Themenheft von „Geschichte und Gesellschaft“, 35 (2009), S.98-134, hier S.99f.
} 
verdichtendes Geflecht von bilateralen Wanderungsverträgen, das diese Wanderungsströme flankierte und strukturierte. So bildete vor dem Ersten Weltkrieg die Arbeitsmigration aus Italien nach Frankreich, Belgien, Luxemburg sowie in die Schweiz und nach Deutschland gemeinsam mit den Wanderungsbeziehungen des deutschen Kaiserreichs zu den österreichischen bzw. russischen Teilen Polens den Kern eines entstehenden europäischen Migrationssystems. Der Erste Weltkrieg führte zur Einbindung der nordafrikanischen Kolonien bzw. Besitzungen Frankreichs (Algerien, Tunesien und Marokko) in den Zusammenhang europäischer Arbeitsmigration, als die massenhafte Rekrutierung von Arbeitskräften und Soldaten in diesen Regionen einsetzte, an die nach 1918 weitere Zuwanderung aus Nordafrika nach Frankreich sowie - wenn auch marginal - nach Belgien anschloss. Im gleichen Kontext stiegen Spanien und Portugal zu wichtigen Migrationspartnern Frankreichs auf und knüpften nach Kriegsende Arbeitsmarktbeziehungen zu weiteren Staaten. Frankreich, Deutschland und die Schweiz blieben auch nach dem Ersten Weltkrieg die Gravitationszentren für Arbeitswanderer aus peripheren Räumen, aber auch Belgien und Luxemburg gewannen als Nachfrager nach Arbeitskräften aus dem Ausland an Bedeutung. In der Zwischenkriegszeit fanden daneben zeitweise Ungarn, Jugoslawien und die Tschechoslowakei sowie andere osteuropäische Staaten einen vertragsbasierten Anschluss an das Migrationssystem. Gleichzeitig erneuerte das wiedervereinigte Polen seine Migrationsbeziehungen zu Deutschland und weitete diese nach Frankreich, Belgien und in den 1930er Jahren auch in die Niederlande aus. Die so bis Kriegsbeginn 1939 gewachsenen Migrationsbeziehungen bestanden größtenteils weiter, als nach dem Ende des Zweiten Weltkriegs und der Deformation des Wanderungssystems durch die deutsche Aggression seine Rekonstruktion einsetzte.

Stärkere Bedeutung erlangten nach 1945 auf der Seite der Zuwanderungsländer Schweden, Österreich und die Niederlande. Gleichzeitig verlagerten sich die Herkunftsgebiete der Arbeitswanderer teilweise nach Südosteuropa, als Griechenland und die Türkei in das Migrationssystem integriert wurden und es Jugoslawien gelang, seine Wanderungsbeziehungen nach Westeuropa zu erneuern. ${ }^{6}$ Den Höhepunkt seiner räumlichen Ausdehnung und der Wanderungsintensität erreichte das Migrationssystem zwischen 1967 und 1972. Allein zwischen 1960 und 1973 erfasste die innereuropäische Arbeitsmigration etwa 30 Millionen Menschen. ${ }^{7}$ Schließlich führte die Wirtschaftskrise der 1970er Jahre zu einem Abbruch dieser Form von Arbeitsmarktbeziehungen. 1973/74 beschlossen alle Anwerbestaaten, ihre Rekrutierungsmaßnahmen zu stoppen. Das auf bilateralen Verträgen fußende europäische Migrationssystem zum temporären Transfer von Arbeitskräften hörte in dieser Form auf zu existieren.

$\mathrm{Zu}$ diesem Zeitpunkt bestanden zwischen den Staaten, die hier in Zusammenhang gebracht werden, mehr als 40 durch bilaterale Wanderungsverträge formalisierte Migrationsbeziehungen. Wie bereits in der Zwischenkriegszeit hatten auch unmittelbar nach dem Ende des Zweiten Weltkriegs erste Staaten Westeuropas auf bilaterale Wanderungsverträge zurückgegriffen, um Zugang zu ausländischen Arbeitsmärkten zu erhalten und die zur Deckung ihres Arbeitskräftebedarfs notwendige Migration zu regulieren. ${ }^{8}$ Zugleich zeigten

\footnotetext{
${ }^{6}$ Hierzu siehe die Länderartikel in Klaus J. Bade/Pieter C. Emmer/Leo Lucassen/Jochen Oltmer (Hrsg.), Enzyklopädie Migration in Europa. Vom 17.Jahrhundert bis zur Gegenwart, Paderborn ${ }^{3} 2010$.

${ }^{7}$ Mark J. Miller/Philip L. Martin, Administering Foreign-Worker Programs, Lexington, MA 1982, S.2. Andere Autoren sprechen von nur etwa 18 Millionen Migranten; Franck Düvell, Europäische und internationale Migration. Einführung in historische soziologische und politische Analysen, Hamburg/ Münster 2006, S.37.

${ }^{8}$ Ein Überblick zu den wichtigsten Wanderungsverträgen der Zwischenkriegszeit findet sich bei Rass, Bilaterale Wanderungsverträge, S.353f.
} 
sich auch erste Abwanderungsländer daran interessiert, in den schwierigen Nachkriegsjahren erneut an den ökonomischen und sozialen Chancen der temporären Arbeitsmigration zu partizipieren. Dies führte bereits in den späten 1940er und frühen 1950er Jahren nicht nur zu einer Wiederbelebung von Wanderungsbeziehungen zu außereuropäischen Zielen, sondern auch zu einer Rekonstruktion des europäischen Migrationssystems. Seinen Kern bildete erneut die Vernetzung Italiens mit seinen traditionell wichtigsten Migrationspartnern, Frankreich, Luxemburg und Belgien, mit denen bis 1951 ebenso Wanderungsverträge zustande kamen wie mit der Schweiz, den Niederlanden, Österreich und Schweden. Westdeutschland war dann der letzte Staat, der die Anwerbung von Arbeitskräften in Italien wieder aufnahm, als 1955 das erste bilaterale Abkommen zwischen beiden Staaten nach 1945 zur Unterzeichnung kam. Anfang der 1960er Jahre setzte dann ein Trend ein, für den die Abkommen Deutschlands mit Spanien und Griechenland als Initialzündung gelten können: Die Ausweitung des Vertragsnetzes nach Süd- bzw. Südosteuropa über Italien hinaus und schließlich nach Nordafrika. Nachdem zunächst Frankreich und Belgien - bereits 1954 bzw. 1957 - diesen Weg durch ihr Engagement in Griechenland eingeschlagen hatten, beschleunigte sich nun die Expansion, befeuert vom Ineinandergreifen anschwellender Wanderungsströme und einer Verdichtung des Vertragsnetzes.

Wanderungsverträge Griechenlands und Spaniens

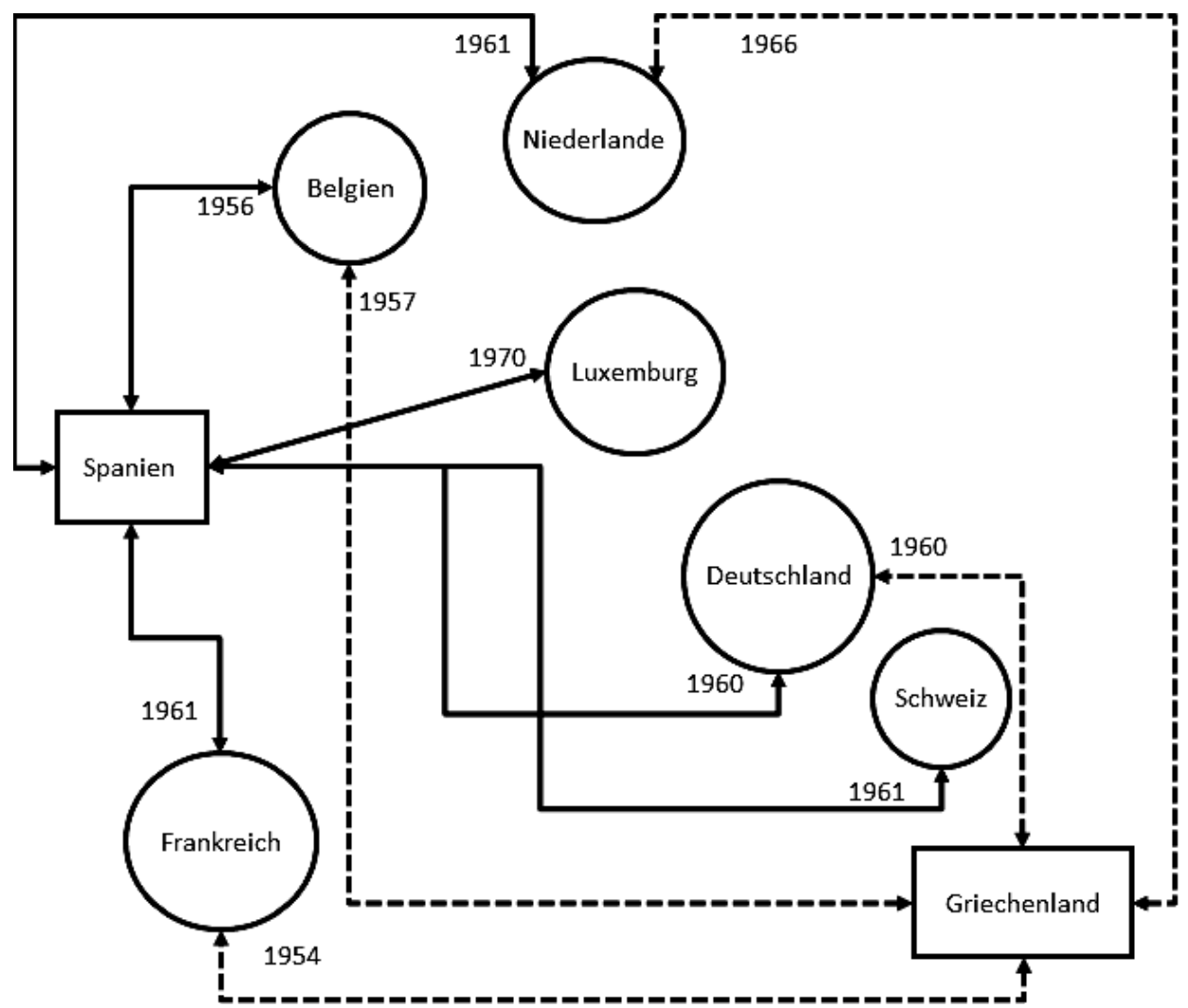


Deutschland übernahm hierbei eine Führungsrolle: Zu den Verträgen von 1960 kamen in den folgenden Jahren Abkommen mit der Türkei, Marokko, Tunesien, Portugal und Jugoslawien hinzu. Ähnlich verhielten sich die übrigen Industriestaaten Westeuropas, die zeitgleich bzw. nacheilend Wanderungsabkommen mit Vertretern dieser Staatengruppe unterzeichneten. Griechenland erschloss sich nach den Abkommen mit Frankreich (1954), Belgien (1957) und Deutschland (1960) durch einen weiteren, jedoch kaum wirksamen Vertrag mit den Niederlanden (1966) den institutionalisierten Zugang zu vier westeuropäischen Arbeitsmärkten. Spanien komplettierte die Riege seiner vertraglich verbundenen Migrationspartner 1961/ 62 in kurzer Folge um Frankreich, die Niederlande, die Schweiz sowie Österreich, im Jahr 1970 dann um Luxemburg. Spätestens in den 1960er Jahren erreichte das europäische Migrationssystem auf diese Weise einen Zustand, in dem jedes Zuwanderungsland mit mehreren Abwanderungsländern und jedes Abwanderungsland mit mehreren Zuwanderungsländern auf Arbeitsmigration konzentrierte Wanderungsbeziehungen unterhielt, wobei sich die Partnerstaaten jeweils weitgehend überschnitten. Das verdeutlicht etwa das Beispiel Deutschland und Frankreich, die sich am Ende des Jahrzehnts alle Migrationspartner bis auf Algerien teilten - darunter auch Spanien und Griechenland. Es ist daher von Bedeutung, die Wechselwirkungen zu berücksichtigen, die sich aus dem Umstand ergaben, dass Wanderungsverhältnisse nicht einfach in der Form 1:1 oder 1:n betrachtet, sondern immer als Teil von n:m Beziehungen gesehen werden müssen, denn auf diesem Arbeitsmarkt stand jeder Nachfrager von Arbeitskraft mit mehreren Anbietern in Beziehung und jeder Anbieter mit mehreren Nachfragern. Dadurch ergaben sich Konkurrenzsituationen um Arbeit oder um Arbeitsplätze, die auch Staaten erfassten, die nicht durch direkte Migrationsbeziehungen oder auf vertraglicher Grundlage aneinander gebunden waren bzw. zur gleichen Kategorie von Marktteilnehmern zählten, also etwa zwischen Frankreich und Deutschland ebenso wie zwischen Griechenland und Spanien.

Kein Akteur dieses internationalen Arbeitsmarktes blieb damit unbeeinflusst vom Handeln seiner Partner und Konkurrenten. Bereits dieses von modellhaften Vorstellungen abgeleitete Argument verdeutlicht, dass unter solchen Bedingungen keine allein auf nationaler Ebene determinierten Migrationsverhältnisse existieren und auch die isolierte Betrachtung bilateraler Konstellationen nicht die tatsächliche Komplexität der Zusammenhänge einfängt. ${ }^{9}$ Im Hinblick auf die Regulierung temporärer Arbeitsmigration folgt aus dieser Erkenntnis die Notwendigkeit, neben den Akteuren auf nationaler Ebene stets auch das Gesamtsystem und die in seinen Strukturen möglichen Wechselwirkungen und Kausalketten im Blick zu halten. Für die Transmission solcher Impulse spielen wiederum bestimmte Institutionen eine wichtige Rolle, wie der bilaterale Wanderungsvertrag oder die Internationale Arbeitsorganisation (ILO) als Standardisierungs- und Kommunikationsplattform. Sie erlaubten es den Marktteilnehmern beispielsweise, ihre Positionen zu definieren bzw. auszuhandeln oder gar zu verbessern, indem sie Risiken und Transaktionskosten minimierten, Interaktionen strukturierten und beschleunigten, politische und ökonomische Interessen koordinierten. ${ }^{10}$

\footnotetext{
9 Ähnlich, wenn auch aus anderer Richtung argumentierend, Paul-André Rosental/David Feldman/ Caroline Douki, Gibt es nationale Migrationspolitik? Über einige Lehren aus den zwanziger Jahren, in: Rudolf von Thadden/Steffen Kaudelka/Thomas Serrier (Hrsg.), Europa der Zugehörigkeiten. Integrationswege zwischen Ein- und Auswanderung, Göttingen 2007, S.69-78.

${ }^{10}$ Rass, Bilaterale Wanderungsverträge, S.104.
} 


\section{Bilaterale Wanderungsverträge}

Bilaterale Wanderungsverträge dienen einem regulierten und zunächst temporären Transfer von Arbeitskraft zwischen zwei Volkswirtschaften. Sie greifen in alle Phasen eines Wanderungsprozesses ein: Ihre Wirkung beginnt mit der selektiven Mobilisierung von Arbeitskräften in deren Herkunftsland, erstreckt sich über deren Transfer zwischen zwei Arbeitsmärkten bis zur Fixierung der Rahmenbedingungen für die Arbeitsmarktpräsenz im Zielland und kann auch Wege für die Aufnahme der Migranten in deren Gesellschaft bzw. Vorkehrungen für ihre Rückwanderung vorzeichnen. ${ }^{11}$

Geboren wurde der moderne Anwerbe- bzw. Wanderungsvertrag - nahezu bereits in seiner endgültigen Gestalt - mit dem Abkommen, das Frankreich und Polen im Jahr 1919 unterzeichneten. ${ }^{12}$ In der Zwischenkriegszeit griff dann die Internationale Arbeitsorganisation die Frage der Verankerung gewisser Mindeststandards hinsichtlich der Anwerbung und des Transfers von Arbeitsmigranten ebenso wie von Arbeitsbedingungen, sozialfürsorglicher und tariflicher Gleichbehandlung oder aufenthaltsrechtlichem Status auf und begann, auf ein Modell derartiger Abkommen hinzuarbeiten, das sich eng am französischen Usus orientierte. Während Wanderungsabkommen in Europa immer gebräuchlicher wurden, bildete sich Ende der 1930er Jahre tatsächlich ein Gerüst derartiger Standards heraus, das die ILO in einem Musterabkommen verankerte. Der Zweite Weltkrieg verhinderte zwar einen faktischen Niederschlag dieser Initiative. Der am Modell der ILO-Konventionen bzw. -Empfehlungen der Jahre 1939/1949 orientierte Wanderungsvertrag sollte in der Nachkriegszeit jedoch beträchtliche Verbreitung und Bedeutung erlangen. ${ }^{13}$

Dass sich der bilaterale Wanderungsvertrag als Instrument zur Verankerung staatlich regulierter Arbeitsmigration in Europa nach 1945 durchsetzte, hatte vor allem zwei Ursachen. Erstens griffen wichtige Akteure, darunter vor allem Frankreich, sehr schnell die Praxis der Zwischenkriegszeit auf, die den Wanderungsvertrag schon einmal zu einer zentralen institutionellen Stütze regulierter grenzüberschreitender Arbeitsmigration gemacht hatte. Die dann immer schnellere Verbreitung des Wanderungsvertrages als Element neu angebahnter bilateraler Wanderungsbeziehungen erhöhte den Druck auf alle Marktteilnehmer, sich dem System solchermaßen verfasster Migrationsbeziehungen anzuschließen. Dies stand, zweitens, in einem engen Zusammenhang mit den Vorteilen dieser Verträge selbst - boten sie doch durch ihre standardisierende Wirkung eine Orientierungslinie für Regulierungsreichweite und -intensität von Arbeitswanderung, die einem „race to the bottom" der Migrationsbedingungen entgegenwirkte und so die für die Arbeitnehmerinnen und Arbeitnehmer in den Zielländern entscheidende Gleichbehandlung von In- und Ausländern zur Vermeidung einer Aushöhlung sozialer und tariflicher Errungenschaften stützte. Dies verband der Wanderungsvertrag mit gewissen Gestaltungsspielräumen für die spezifischen migrationspolitischen Präferenzen der beteiligten Staaten. Gleichzeitig gewährten die in Wanderungsverträgen getroffenen Vereinbarungen den Migranten selbst gewisse, zumindest auf dieser Ebene gesicherte Rahmenbedingungen und ihren Herkunftsstaaten ihrerseits Eingriffs- und Steuerungsmöglichkeiten. Kombiniert führten beide Eigenschaften dazu, dass sich zumindest normativ abgesicherte Mindeststandards regulierter Arbeitsmigration durchsetzten. Ferner begünstigten die Wanderungsverträge die Transparenz des Wett-

\footnotetext{
11 Ders., Institutionalisierungsprozesse, S.296f.

${ }^{12}$ Zur Vorgeschichte des Wanderungsvertrages Madeleine Herren, Internationale Sozialpolitik vor dem Ersten Weltkrieg, Berlin 1990, S.140f.

${ }^{13}$ Die Empfehlungen 61 und 62 sowie das Übereinkommen 66 aus dem Jahr 1939 sowie die Empfehlung 86 und das Ubereinkommen 97 aus dem Jahr 1949 finden sich auf der Website der ILO unter http://www.ilo.org/ilolex/german/docs/.
} 
bewerbs, in dem für alle Marktteilnehmer die Bedingungen, zu denen sich jeweils zwei Migrationspartner über die Zulassung von Arbeitsmigration verständigt hatten, durch die Wanderungsabkommen und die ihnen in der Regel beigefügten Musterarbeitsverträge offenlagen. ${ }^{14}$ Das Ergebnis war ein doppelter Konvergenzprozess, in dem sich immer mehr Staaten dem aus bilateralen Beziehungen entstehenden multipolaren internationalen Arbeitsmarkt anschlossen, während sich die in den Verträgen angebotenen Migrationsbedingungen durch Wettbewerbseffekte tendenziell anglichen, bisweilen aber auch deutlich in die eine oder andere Richtung ausschlugen. Agierte ein anwerbender Staat monopolartig auf dem Arbeitsmarkt eines Abwanderungslandes, wie beispielsweise Deutschland zeitweise in Griechenland oder der Türkei, beeinflusste die Konkurrenz um die nur von ihm gebotenen Migrationschancen die Bedingungen zu seinen Gunsten. Konkurrierten dagegen mehrere Staaten um die Arbeitswanderer eines Landes, so führte dies zu einer Verbesserung der ihnen gebotenen Wanderungsbedingungen. ${ }^{15}$

\section{Die Integration Griechenlands und Spaniens in das Vertragssystem}

Was lässt sich aus diesen übergeordneten Betrachtungen im Hinblick auf die Positionierung Griechenlands und Spaniens im europäischen Migrationssystem durch den Abschluss bilateraler Wanderungsabkommen und insbesondere für ihr Verhältnis zu Deutschland ableiten? Obgleich die saisonale Wanderung spanischer Landarbeitskräfte nach Frankreich bereits ab den 1830er Jahren nachweisbar ist, spielten europäische Wanderungsziele für das Land lange Zeit eine untergeordnete Rolle. ${ }^{16}$ Erst die Rekrutierung spanischer Arbeiter für die französische Kriegswirtschaft im Ersten Weltkrieg und eine sich in der Zwischenkriegszeit ausweitende Bedeutung der Arbeitswanderung nach Frankreich änderten dies und deuteten eine langsame Verschiebung der Wanderungsströme an. ${ }^{17} \mathrm{Zu}$ einem erstrangigen Wanderungsziel spanischer Arbeitsmigranten stiegen europäische Länder allerdings erst nach dem Zweiten Weltkrieg auf. ${ }^{18}$ In den ersten Jahren nach 1945 erlebte Spanien zunächst eine Wiederbelebung der Migrationsbeziehungen zu Südamerika, während das Regime Francos an seinem wirtschaftspolitischen Autarkiekurs festhielt. ${ }^{19}$ Im Verlauf der 1950er Jahre zeigte sich jedoch, dass diese Abschottung weder politisch noch ökonomisch durchzuhalten war. Das vollkommene Scheitern nicht nur der wirtschaftlichen Isolierung, sondern auch der Versuche, Anfang der 1960er Jahre die Wiedereingliederung in die Weltwirtschaft einzuleiten, zeitigten gravierende soziale Folgen und steigerten den ohnehin durch eine wanderungsfeindliche Politik rückgestauten Auswanderungsdruck in Spanien

\footnotetext{
${ }^{14}$ Rass, Institutionalisierungsprozesse, S.299f.

${ }^{15}$ Ebenda, S.473f.

${ }^{16}$ Joseph Farré, L'émigration espagnole vers l'Europe pendant la deuxième moitié du XX $\mathrm{XX}^{\mathrm{e}}$ siècle. Remarques sur le phénomène de l'émigration et de la recherche, in: Paul Vaiss/Joseph Farré/Klaus Morgenroth (Hrsg.), Les migrations du travail en Europe, Frankfurt a.M. 2003, S.135-154; Horst Pietschmann, Spanien und Portugal, in: Bade/Emmer/Lucassen/Oltmer (Hrsg.), Enzyklopädie Migration in Europa, S.220-242, hier S.238.

${ }^{17}$ Pierre Ewenczky, Les Espagnoles. Emigration et développement économique, in: Mohamed Bennabou/Jean-Pierre Garson/Georges Tapinos (Hrsg.), L’Argent des immigrés. Revenus, épargne et transferts de huit nationalités immigrées en France, Paris 1981, S.71-98, hier S.74.

${ }_{18}$ Bürgerkrieg und Sieg der Faschisten hatten Fluchtbewegungen vorübergehend vorherrschend werden lassen; während des Zweiten Weltkriegs kam es dann zu einer quantitativ wenig bedeutenden Anwerbung spanischer Arbeitskräfte für die deutsche Kriegswirtschaft; Félix Santos Delgado, Exiliados y emigrados 1939-1999, Madrid 1999.

${ }^{19}$ Axel Kreienbrink, Einwanderungsland Spanien. Migrationspolitik zwischen Europäisierung und nationalen Interessen, Frankfurt a.M. 2004, S.47f.
} 
weiter. ${ }^{20}$ Entsprechend gewann eine regulierte temporäre Abwanderung von Arbeitskräften in den spanischen Wirtschaftsplanungen zu Beginn der 1960er Jahre erheblich an Gewicht. ${ }^{21} \mathrm{Zu}$ diesem wirtschafts- und wanderungspolitischen Kurswechsel passte die Entwicklung auf der politischen Ebene, als es Franco gelang, die Ächtung seines Landes in Europa aufzubrechen und sich den USA als Verbündeter im Kalten Krieg anzudienen. ${ }^{22}$ Förderung, aber auch Kontrolle von Arbeitswanderung avancierte in Spanien zu diesem Zeitpunkt zu einem wichtigen Politikfeld. ${ }^{23}$ Die normativen und institutionellen Voraussetzungen für die Umsetzung eines solchen Programms waren bereits 1956 mit der Gründung einer Auswanderungsbehörde, des Instituto Español de Emigración, sowie der Verabschiedung eines Auswanderungsgesetzes gegeben, das die Grundlagen für eine strikte Kontrolle grenzüberschreitender Migration schuf und 1960 bzw. 1962 durch weitere Normen ergänzt wurde. Arbeitsmigration erfuhr nun eine aktive staatliche Förderung und galt als ökonomische wie soziale Chance für die Abwanderer und ihre Familien mit positiven Auswirkungen für das Gemeinwohl. Die Ausübung des den Spaniern gewährten Grundrechts auf Auswanderung unterwarf das franquistische Regime allerdings strikter staatlicher Kontrolle. Wem die Auswanderungsbehörde jedoch die Möglichkeit zur Arbeit im Ausland eröffnete, der sollte weitreichende Unterstützung und Hilfestellungen erfahren. ${ }^{24}$

Deutschland erlebte im Gefolge des Anwerbevertrages von 1960 nach der Arbeitsmigration während des Zweiten Weltkriegs erneut eine Arbeitskräfteeinwanderung aus Spanien. Im Gegensatz zur Migration nach Frankreich, bei der das Zusammenwirken von geographischer Nähe und französischem Laisser-faire hinsichtlich der illegalen Zuwanderung spanischer Arbeiterinnen und Arbeiter zu einem hohen Anteil unregulierter Migration führte, verlief die Arbeitswanderung aus Spanien nach Deutschland in hohem Maß über die von der spanischen Auswanderungsbehörde und der deutschen Arbeitsverwaltung kontrollierten Migrationskanäle. Der regulierte Teil der Migrationsbewegung erfasste bis zu 70 Prozent der spanischen Einwanderer, und Deutschland fungierte zeitweise als das wichtigste Aufnahmeland für spanische Dauerarbeitskräfte, die im sekundären Sektor Beschäftigung fanden. ${ }^{25}$ Den quantitativen Bedeutungsunterschied zu Frankreich verdeutlicht indes die Zahl der spanischen Einwohner Deutschlands am Ende des Betrachtungszeitraumes. Zwischen 1960 und 1969 wanderten 444119 Spanier nach Deutschland, nach dem Ende der Anwerbung im Jahr 1974 lebten hier jedoch nur noch 272676 von ihnen. ${ }^{26}$ Mit einem Anteil von 6,6 Prozent der ausländischen Wohnbevölkerung bildeten sie nach

\footnotetext{
${ }^{20}$ Pablo Antolín Nicolás, International Migration Flows. The Case of Spain (1960-1988), Oxford 1994, S.2f.; Walther L. Bernecker, Sozialgeschichte Spaniens im 19. und 20.Jahrhundert, Frankfurt a.M. 1991, S.298f.

${ }^{21}$ Francisco Comin/Pablo Martin Aceña, Industrial Planning in Spain under the Franco Regime, in: Pablo Martín Aceña/James Patrick Simpson (Hrsg.), The Economic Development of Spain since 1870, Aldershot 1995, S.390-401, hier S.395f.

${ }^{22}$ Walther L. Bernecker, Geschichte Spaniens im 20.Jahrhundert, München 2010, S.226f.; JohannesDieter Steinert, Migration und Politik. Westdeutschland - Europa - Übersee 1945-1961, Osnabrück 1995, S.290f.; Klaus Hommel, Der Beginn der wirtschaftlichen Westintegration Spaniens durch die Beitritte zur OEEC, zum IWF und zur Weltbank, in: Jürgen Elvert/Michael Salewski (Hrsg.), Historische Mitteilungen der Ranke-Gesellschaft, Stuttgart 1992, S.253-272.

${ }^{23}$ Marion Bernitt, Die Rückwanderung spanischer Gastarbeiter. Der Fall Andalusien, Königstein i.Ts. 1981, S.8f.

${ }^{24}$ Carlos Sanz Díaz, Las relaciones del IEE con Alemania, in: Luís M. Calvo Salgado u. a., Historia del Instituto Español de Emigración. La política migratoria exterior de España y el IEE del Franquismo a la transición, Madrid 2009, S.167-187; siehe hierzu auch die Beiträge von Axel Kreienbrink und Carlos Sanz Díaz in dem vorliegenden Band.

${ }^{25}$ Juan Bautista Vilar/Maria José Vilar, La emigración española a Europa en el siglo XX, Madrid 1999, S.64f.

${ }^{26}$ Walther L. Bernecker, Spanien-Handbuch. Geschichte und Gegenwart, Tübingen 2006, S.312.
} 
türkischen, italienischen und jugoslawischen Migranten bereits eine der kleineren Gruppen. ${ }^{27}$ In Frankreich stellten Spanier sowohl in absoluten als auch in relativen Werten eine deutlich wichtigere Einwandererminorität. Dieses Ergebnis kam jedoch nur auf der Grundlage der wesentlich älteren Wanderungsbeziehungen zwischen beiden Staaten zustande, hatten in Frankreich doch bereits zu Beginn der 1930er Jahre rund 350000 Spanier gelebt. Im Verlauf der 1960er Jahre offenbarte sich indes Überraschendes: Mit 426119 Migranten lag die Zahl der sich nun nach Frankreich wendenden Spanier geringfügig niedriger als die Zahl derer, die nach Deutschland gingen. Zumindest vorübergehend und nicht zuletzt mit Hilfe des bilateralen Wanderungsvertrages und der sich an ihn knüpfenden starken Regulierung der Anwerbung bzw. Migration zwischen beiden Staaten - war es Deutschland gelungen, Frankreich den ersten Rang unter den Zielländern der spanischen Arbeitswanderung zu nehmen. ${ }^{28}$

Die Entwicklung Griechenlands seit seiner Unabhängigkeit vom Osmanischen Reich 1828/29 scheint von Faktoren bestimmt, die das Land bereits früh zu einer Quelle vielfältiger Abwanderungsbewegungen machten. Das Ausbleiben einer Industrialisierung, eine rückständige Landwirtschaft, daraus resultierende Abwanderung aus den ländlichen Distrikten und wachsende soziale Spannungen, die Balkankriege vor dem Ersten Weltkrieg, der Konflikt mit der Türkei in den 1920er Jahren, die anhaltende ökonomische Perspektivlosigkeit im Land und schließlich die deutsche Besatzung und der auf die Befreiung folgende griechische Bürgerkrieg sorgten für einen latent hohen Migrationsdruck. Dieser entlud sich schon vor dem Zweiten Weltkrieg in einer Massenauswanderung aus Griechenland vor allem in die Vereinigten Staaten von Amerika, aber auch in andere überseeische Zielregionen. ${ }^{29} \mathrm{Zu}$ Beginn der 1950er Jahre hatten wirtschaftliche Rückständigkeit und Kriege über fast 100 Jahre die griechische Migrationsgeschichte geprägt. Durch die Massenauswanderung entstanden dichte Migrationsbeziehungen vor allem zu den USA, zu Kanada und zu Australien, die auch in der Nachkriegszeit primäre Ziele griechischer Migranten blieben, sodass noch 1953 ein Anteil von 85 Prozent der Abwanderung auf Ziele in Übersee entfiel. ${ }^{30}$ Griechenland konnte zwar bereits in den 1950er Jahren Anwerbeabkommen mit Belgien und Frankreich abschließen, jedoch kaum davon profitieren, hatten beide für die Anwerbestaaten doch eher strategischen Charakter bzw. lassen sich in der politischen Anbindung an Westeuropa nach dem Ende des Bürgerkriegs verorten. ${ }^{31}$ Im Jahr 1960 folgte

\footnotetext{
${ }^{27}$ Gonzalo Garcia Passigli, Report on Spanish Emigration, in: Ayse Kudat/Yilmaz Özkan (Hrsg.), The Comparative Study of the Re-integration Policy of Five European Labor Exporting Countries, Berlin 1975, S.73-119, hier S.7; Carlos Sanz Díaz, España y la Republica Federal de Alemania (1949-1966): Política, Económica y emigración, entra la Guerra Fría y la destentión, Madrid 2005, S.955f.

${ }^{28}$ Bernecker, Spanien-Handbuch, S.312. Solche Befunde stehen selbstverständlich immer unter dem Vorbehalt unvollständiger statistischer Informationen, die einen Teil der Wanderungsbewegung ausblenden, der sich der statistischen Erfassung der einen oder anderen Seite entzog; Carlos Sanz Díaz, „Illegale“, „Halblegale“, „Gastarbeiter“. Die irreguläre Migration aus Spanien in die Bundesrepublik Deutschland im Kontext der deutsch-spanischen Beziehungen 1960-1973, Berlin 2010, S. 15 f.; Kreienbrink, Einwanderungsland, S.51.

${ }^{29}$ Demetrios Papademetriou, Illusions and Reality in International Migration. Migration and Development in Post World War II Greece, in: International Migration 23 (1985), S.211-223, hier S.213; Konstantinos Aidinidis, Zur griechischen Arbeitsemigration unter besonderer Berücksichtigung der Remigrationsproblematik. Eine empirische Untersuchung 1990, S.23f.

${ }^{30}$ Schätzungen sprechen davon, dass im gesamten Zeitraum zwischen 1821 und 1854 insgesamt 85,3\% aller griechischen Auswanderer in die USA gegangen seien; Loukia M. Moussourou, La Sociologie de la Migration en Grèce depuis 1960, in: Current Sociology 32 (1984), S.89-121, hier S.90.

${ }^{31}$ Pavlos Tzermias, Politik im neuen Hellas. Strukturen, Theorien und Parteien im Wandel, Tübingen 1997, S.83f. Die Bewegung griechischer Migranten in den belgischen Bergbau flaute gar nach Abschluss des griechisch-belgischen Abkommens ab.
} 
dann als der im Hinblick auf die resultierende Wanderungsdynamik bedeutendste Wanderungsvertrag Griechenlands jener mit Westdeutschland. ${ }^{32}$

Diese Aufnahme formeller Migrationsbeziehungen zwischen Griechenland und Deutschland im Jahr 1960 hatte eine mehrjährige Vorgeschichte. Im Unterschied zur Anbahnung des Vertragsabschlusses mit Belgien rührten solche Aktivitäten jedoch nicht von Bemühungen her, die ansteigende informelle Vermittlung griechischer Arbeitswanderer zu kanalisieren, sondern lag auf der politischen Ebene. Bereits ab 1955 mahnten griechische Diplomaten in Deutschland die Aufnahme von Arbeitsmarktbeziehungen unter Verweis auf die Opfer und Schäden an, die das Land unter deutscher Besatzung erlitten hatte. Als die griechische Regierung ihre Anstrengungen verstärkte, den Wiederaufbau und die Entwicklung des Landes mit Hilfe von Wirtschaftsplänen voranzutreiben, und für die Periode zwischen 1960 und 1964 ihren ersten Entwicklungsplan ausarbeitete, gewann die Abwanderung von Arbeitskräften als Entlastungsventil und Devisenbeschaffer immer größere Bedeutung. ${ }^{33}$ Nach anfänglicher Zurückhaltung der deutschen Seite, die sich zunächst nicht an einer Ausweitung der Anwerbung ausländischer Arbeitskräfte interessiert zeigte, gab die wachsende Nachfrage nach ausländischen Arbeitskräften durch die deutsche Wirtschaft den Ausschlag. ${ }^{34}$ Als die Bundesrepublik ihre Migrationsbeziehungen in Richtung Spanien ausbaute, kamen parallel auch Verhandlungen mit Griechenland in Gang, die 1960 ihren Abschluss fanden. Ein nicht unbedeutender Katalysator dürfte dabei die sich anbahnende Bindung Griechenlands an die Europäische Wirtschaftsgemeinschaft (EWG) gewesen sein, die sich im Juli 1961 durch einen Assoziierungsvertrag manifestierte. ${ }^{35}$

Wie groß der Abwanderungsdruck in Griechenland mittlerweile war und wie schnell unter diesen Bedingungen ein durch Rekrutierung amplifizierter Migrationsprozess seine Dynamik entfalten konnte, zeigt die Entwicklung der deutsch-griechischen Arbeitskräftewanderung. ${ }^{36}$ Schon unmittelbar mit der ersten deutschen Rekrutierungskampagne setzte ein Ansturm Tausender griechischer Auswanderer auf die Rekrutierungsbüros in Athen und Saloniki ein, die sich, getrieben von extremer sozialer Ungleichheit, hoher Arbeitslosigkeit und ausbleibenden Wohlstandszuwächsen, für die Abwanderung entschieden. Im Vergleich zum Jahr 1959 verzehnfachte sich 1960 die Zahl der Griechen, die nach Deutschland zuwanderte, auf 21500, um im Folgejahr auf über $50000 \mathrm{zu}$ wachsen und 1965 mit 80500 Abwanderern ihren Höhepunkt zu erreichen. Die Arbeitswanderung überholte im Verlauf des Jahrzehnts, vor allem getrieben von der Massenwanderung nach Westdeutschland, sogar das Bevölkerungswachstum, sodass die Bevölkerung Griechenlands zu schrumpfen begann. ${ }^{37}$ Gleichzeitig partizipierten andere Migrationsziele nicht nur relativ, sondern auch in absoluten Zahlen immer weniger stark an der griechischen Abwanderung. Vom Beginn der Anwerbung bis zu ihrer Einstellung im Jahr 1973 kamen 615400 griechische Arbeitnehmer und ihre Familienangehörigen nach Deutschland, von denen etwa die Hälfte im gleichen Zeitraum wieder nach Griechenland zurückkehrte. ${ }^{38}$ Mehr als 60 Prozent

\footnotetext{
${ }^{32}$ Hinzu kam dann 1966 nur noch das Abkommen mit den Niederlanden, das letztlich ohne bedeutende Auswirkung auf die griechischen Wanderungsströme blieb.

${ }^{33}$ Richard Clogg, Geschichte Griechenlands im 19. und 20.Jahrhundert, Köln 1997, S.183f.; Eleni Andrikopoulou/Grigoris Kafkalas, Greek Regional Policy and the Process of Europeanization 19612000, in: Dionyssis G. Dimitrakopoulos/Argyris G. Passas (Hrsg.), Greece in the European Union, Bd.2, London 2004, S.35-47, hier S.37f.

${ }^{34}$ Rass, Institutionalisierungsprozesse, S. 75.

35 Tzermias, Politik im neuen Hellas, S.90; das Abkommen trat Anfang November 1962 in Kraft.

${ }^{36}$ Manuel Gogos, Überblendungen. Deutsche Besatzung in Griechenland und die griechische Arbeitsmigration nach Deutschland, in: Projekt Migration, Köln 2005, S.822f.

37 Tzermias, Politik im neuen Hellas, S.92.

38 Theodore P. Lianos, Flows of Greek Out-migration and Return-migration, in: International Migration 18 (1975), S.119-133, hier S.122.
} 
der griechischen Arbeitskräfte wurden über die deutschen Rekrutierungsstellen angeworben bzw. namentlich angefordert, die übrigen wählten den Weg über die deutschen Konsulate oder die Einreise mit einem Touristenvisum. ${ }^{39}$ Deutschland nahm in den zwölf Jahren der Arbeitskräfterekrutierung in Griechenland rund 85 Prozent aller griechischen Auswanderer auf, die innerhalb Europas blieben, sodass zu Beginn der 1970er Jahre für kurze Zeit etwa 11,5 Prozent der griechischen Bevölkerung in Deutschland lebten. ${ }^{40}$ Gemessen an der überragenden Bedeutung Deutschlands als Migrationspartner Griechenlands nach dem Zweiten Weltkrieg blieben die Auswirkungen weiterer bilateraler Anwerbeabkommen bzw. Migrationsbeziehungen zweitrangig. ${ }^{41}$

In beiden Fällen kam der Vereinbarung mit Deutschland also eine Art Achsenfunktion zu. Spanien leitete mit dem Vertrag von 1960 eine Diversifikation seiner Vertragsbindungen ein, in deren Verlauf auch Frankreich 1961 einer stärkeren Regulierung der Arbeitsmigration zwischen beiden Staaten zustimmte. Für Frankreich musste, wie die modellhaften Betrachtungen gezeigt haben, der Stellenwert eines Wanderungsvertrages in dem Maß steigen, wie Spanien auf Migrationsbeziehungen mit Dritten verweisen konnte, die sich dieser Institution bedienten und sich damit in Richtung auf regulierte Anwerbung und standardisierte Migrationsbedingungen bewegten. Spanien indes näherte sich dem erklärten Ziel seiner neuen Wanderungspolitik, Arbeitsmigration stärker steuerbar zu machen und der Kontrolle der Auswanderungsbehörde zu unterwerfen. Gerade im Hinblick auf die alten und traditionell weitgehend unkontrollierbaren Migrationsverhältnisse gegenüber Frankreich versprach ein Wanderungsvertrag zumindest auf der normativen Ebene die gewünschte Transformation der Wanderungsbeziehungen. Spanien entwickelte bis zum Anwerbestopp ein Netz von sechs vertraglich verbundenen Anwerbestaaten, mit denen es beim Aushandeln und Verteilen von Migrationschancen operieren konnte. ${ }^{42}$

Für Griechenland erwies sich der Vertrag mit Westdeutschland als entscheidendes Moment für die Öffnung des europäischen Arbeitsmarktes, denn er begründete die erste innereuropäische Wanderungsbeziehung des Landes, die sich nicht mehr im Bereich geduldeter individueller Zuwanderung bzw. privater Anwerbeoperationen bewegte oder aus einem Wanderungsvertrag ohne mit ihm verbundene signifikante Wanderungsbewegung bestand. Diese erwies sich als so dynamisch, dass Griechenland bis zum Anwerbestopp weder institutionell noch quantitativ nach einer vergleichbaren Ausweitung seiner Wanderungsoptionen strebte. Das Modell des bilateral verfassten europäischen Arbeitsmarktes legt allerdings die Vermutung nahe, dass der Preis für diese starke Abhängigkeit Griechenlands von den aus Westdeutschland gebotenen Wanderungschancen ein Verlust von Gestaltungsmacht hinsichtlich der Beeinflussung von Migrationsstandards war. ${ }^{43}$ Die Verbindungen beider Staaten im europäischen Arbeitsmarkt zeigen Spanien also als einen multipel vernetzten und Griechenland als einen zwar institutionell mehrfach gebundenen, tatsächlich jedoch auf ein Partnerland fixierten Akteur.

\footnotetext{
${ }^{39}$ Nicholas P. Glytsos/Louka T. Katseli, Greek Migration. The Two Faces of Janus, in: Klaus F. Zimmermann (Hrsg.), European Migration. What do we know?, Oxford 2005, S.337-388, hier S.352.

40 Theodore P. Lianos, Greece. Waning of the Labor Migration, in: Daniel Kubat (Hrsg.), The Politics of Migration Policies. Settlement and Integration - the First World into the 1990s, New York 1993, S. 249-261, hier S.250; Nicholas P. Glytsos/Louka T. Katseli, Theoretical and Empirical Determinants of International Labour Mobility. A Greek-German Perspective, in: Anthony Philip Thirlwall/Ian R. Gordon (Hrsg.), European Factor Mobility. Trends and Consequences, Basingstoke 1989, S.95-115, hier S.97. Bezogen auf die gesamte Auswanderung aus Griechenland lag der Anteil Deutschlands bei $56 \%$, bleiben die Krisenjahre 1967/68 ausgeklammert, bei 60 \%; Moussourou, La Sociologie, S.95.

${ }^{41}$ Lianos, Greece, S. 255.

${ }^{42}$ Rass, Institutionalisierungsprozesse, S.249f.

${ }^{43}$ Ebenda, S.163f.
} 


\section{Die Wanderungsverträge Spaniens und Griechenlands mit Westdeutschland}

Wie ordnen sich das spanisch-deutsche bzw. das griechisch-deutsche Wanderungsabkommen in die europäische Vertragslandschaft der 1960er Jahre ein? Zunächst zeigt ein Vergleich der Vertragstexte, dass sich beide Übereinkommen stark ähneln und in ihren Grundlinien den Vorgaben der ILO folgen. ${ }^{44}$ Als Erklärung hierfür scheint plausibel, dass sie aus einer Phase datieren, in der Westdeutschland auf eine schnelle Ausweitung des Zustroms ausländischer Arbeitskräfte hinarbeitete und daher die Verwendung der ILO-Richtlinien, die nicht zuletzt auch im Interesse der Abwanderungsländer lag, als Vehikel für eine schnelle Vernetzung nutzte. Diese Interpretation wird auch dadurch gestützt, dass Spanien und Griechenland keine Neulinge im Beziehungsgeflecht mehr waren, sondern ihre Erfahrungen aus sowohl vertraglich verfassten als auch noch nicht formalisierten Wanderungsbeziehungen in die Verhandlungen einbringen konnten. ${ }^{45}$

Signifikant dagegen sind allerdings sowohl die Unterschiede zum bereits bestehenden deutsch-italienischen Abkommen als auch das Verhältnis der beiden Verträge zu den jeweils anderen Abkommen der beiden Abwanderungsländer. Deutschland führte mit den beiden Wanderungsabkommen des Jahres 1960 eine Innovation in die Anwerbeverträge ein: das Listenverfahren. Es erlaubte dem Partnerstaat, unabhängig von den Anforderungen deutscher Arbeitgeber Bewerberpools zu bilden, aus denen eingehende Stellenangebote besetzt werden konnten. Zu diesem Zweck konnten sich Abwanderungswillige bei der in Griechenland bzw. Spanien jeweils für die Durchführung der Anwerbung zuständigen Behörde melden. Dort durchliefen sie bereits den Selektionsprozess der entsendestaatlichen Seite, also vor allem eine erste Gesundheitsuntersuchung. Ferner konnte geprüft werden, ob die Kandidaten die arbeitsmarkt- oder sozialpolitischen Voraussetzungen für die Zulassung zum Anwerbeverfahren erfüllten, also beispielsweise arbeitslos waren und kein Qualifikationsprofil aufwiesen, das sie - um den Facharbeitermangel im Inland nicht zu vergrößern - von der regulierten Arbeitsmigration ausschloss. Ebenfalls möglich war eine Überprüfung, ob die potentiellen Abwanderer bereits ihren Militärdienst absolviert hatten. Da das Anwerbeverfahren grundsätzlich mit einer beidseitigen Selektion, also durch das Entsendeland und den anwerbenden Staat verbunden sein sollte, und beide unter Umständen unterschiedliche Kriterien anlegten, verhieß das Listenverfahren eine Beschleunigung des Vorgangs und eine bessere Planbarkeit der Nachfragebefriedigung. Zudem bot die Größe des Bewerberpools einen Indikator für das verfügbare Arbeitskräftepotential. Diesen Effekt nutzte vor allem Deutschland, um die Anforderungen ausländischer Arbeitskräfte, die bei der Arbeitsverwaltung eingingen, strategisch auf die deutschen Anwerbestellen in den verschiedenen Staaten zu verteilen. Es erlaubte den Entsendestaaten im Gegenzug, schon im Vorfeld der tatsächlichen Anwerbung politische Effekte über die regionale Verteilung der Chance auf Partizipation am Wanderungsprozess zu erzielen, da man bereits diese VerheiBung einer Chance auf Arbeitswanderung in den Abwanderungsländern vielfach als erfolgreiches sozial- bzw. wirtschaftspolitisches Handeln von Regierungen bzw. ihren Politikern und Bürokratien wahrnahm.

\footnotetext{
${ }^{44}$ So verweist zwar Kreienbrink zur Stützung seiner Interpretation, die Anwerbeverträge spiegelten vor allem den Gestaltungswillen der Anwerbestaaten, darauf, der deutsch-spanische Vertrag von 1960 enthalte im Gegensatz zum spanisch-französischen Abkommen keine Regelungen für einen Familiennachzug, was den Empfehlungen der ILO widersprochen hätte, übersieht jedoch Artikel 17 der deutsch-spanischen Vereinbarung vom 29.3.1960; Kreienbrink, Einwanderungsland, S.62, Anm.164.

${ }^{45}$ Sanz Díaz, „Illegale“; Lina Venturas, The Beginning of Greek Post-war Emigration to Belgium. Networks and Strategies, in: Evangelos Konstantinou (Hrsg.), Griechische Migration in Europa. Geschichte und Gegenwart, Frankfurt a.M. 2000, S.217-226.
} 
Gleichzeitig waren die Abkommen sowohl für Griechenland - klammert man den ganz und gar unattraktiven griechisch-französischen Vertrag von 1954 aus - als auch für Spanien die ersten, die nicht nur den Bergbau des Ziellandes für Arbeitswanderer öffneten, sondern deren gesamten Arbeitsmarkt. So hatte Spanien zwar 1956 ein Abkommen mit Belgien erzielt, das neben einem Schnellverfahren für die Anwerbung auch weitreichende Möglichkeiten für Lohnüberweisungen und, so wie es die Vorgaben der ILO forderten, die Beseitigung aller Freizügigkeitsbeschränkungen nach fünf Jahren Aufenthalt beinhaltete. Gleiche Regelungen enthielt auch der belgisch-griechische Vertrag aus dem Folgejahr. Beide Abkommen erlaubten jedoch die Anwerbung nur für den belgischen Steinkohlenbergbau. ${ }^{46}$ Das deutsche Angebot im Jahr 1960 schien daher ungleich attraktiver: Zugang zum gesamten Arbeitsmarkt für ein breiteres Alterssegment bei relativ guten Verdienstmöglichkeiten, allerdings um den Preis eines stärker reglementierten Aufenthaltsregimes. Die weitere Entwicklung in der Vertragslandschaft deutet auf die Auswirkungen solcher Verbesserungen jenseits der direkt von ihnen definierten Migrationsbeziehungen hin. Belgien etwa verzichtete in seinen Verträgen mit Marokko, der Türkei und mit Tunesien auf eine Beschränkung der Erstanwerbung auf den Steinkohlenbergbau und öffnete stattdessen seinen gesamten Arbeitsmarkt. Dies geschah einerseits unter dem Druck der Nachfrage nach ausländischen Arbeitnehmerinnen und Arbeitnehmern, die auch in Belgien im Verlauf der 1960er Jahre stark wuchs. Andererseits ließen sich solche Einschränkungen angesichts eines immer breiteren Spektrums potentieller Wanderungsziele, die bessere Zugangsbedingungen zu ihren Arbeitsmärkten boten, nur noch unter Inkaufnahme sinkender Anwerbezahlen aufrechterhalten.

\section{Anwerbeabkommen und Migrationsregime der Entsendeländer}

Spanien betrachtete am Ende der 1950er Jahre, ebenso wie Griechenland und die Mehrzahl der Abwanderungsländer der europäischen Peripherie, die temporäre Entsendung von Arbeitskräften ins Ausland als einen Hebel für die Entwicklung seiner Wirtschaft. Die Entlastung des heimischen Arbeitsmarktes, die Milderung sozialer Probleme und der Zufluss von Devisen aus Lohnüberweisungen sowie die Vorstellung, heimkehrende Migranten würden wertvolle berufliche Qualifikationen mitbringen, charakterisieren das Motivkonglomerat, das diese wirtschafts-, sozial- und arbeitsmarktpolitische Vorstellung stützte. ${ }^{47}$

Die Umsetzung dieses Anspruches gelang in Spanien auf der Grundlage langer Erfahrungen als Auswanderungsland und gestützt auf die autoritären Strukturen eines korporatistischen Staates nicht nur auf der politischen bzw. diplomatischen, sondern auch auf der praktischen Ebene zumindest partiell. Das Instituto Español de Emigración verfolgte bei seiner Zusammenarbeit mit der deutschen Arbeitsverwaltung eine Politik strikter Abwanderungskontrolle, die ab 1960 auf entsprechenden Ergänzungen der Wanderungsgesetze basierte. Entsprechend lehnten die spanischen Behörden jede nicht-steuerbare Form individueller Migration, etwa mit Touristenvisum nach Frankreich oder auf dem sogenannten Zweiten Weg nach Deutschland grundsätzlich ab. ${ }^{48}$ Das Ziel dieses Ansatzes war es, die Anwerbung so zu steuern, dass sie vor allem Regionen Spaniens mit schlechter Arbeitsmarktlage entlastete, ohne diesen Gebieten zugleich wertvolle Facharbeitskräfte zu rauben. ${ }^{49}$

\footnotetext{
${ }^{46}$ Amalia Alexiou, L'immigration greque en Belgique, in: Anne Morelli (Hrsg.), Histoire des étrangers et de l'immigration en Belgique de la préhistoire à nos jours, Brüssel 1992, S.274-289.

${ }_{47}$ Bade, Europa, S.315.

48 Sanz Díaz, Las relaciones del IEE con Alemania.

${ }^{49}$ Ders., „Illegale“, S.25f.
} 
Ein spezifisches Element der daraus resultierenden Anwerbeverfahren bestand in der Zuweisung der Anwerbekommissionen Deutschlands, Frankreichs oder anderer Vertragspartner Spaniens zu bestimmten Zonen. Dieses Vorgehen beeinflusste durchaus die Zusammensetzung der von Spanien ausgehenden Wanderungsströme, lief allerdings der Zielwahl vieler Migranten entgegen, sodass solche rigiden Steuerungsversuche auch zu einem Motor individueller und zeitweise illegalisierter Abwanderung wurden. ${ }^{50}$ So blieb es illusorisch, unregulierte Arbeitsmigration gänzlich zu unterbinden, solange der Arbeitskräftebedarf in den anwerbenden Staaten diese zu einer großzügigen Zulassungspolitik motivierte. ${ }^{51}$ Vor allem nach Frankreich kamen nur 20 bis 40 Prozent der Arbeitskräfte auf dem von der spanischen Auswanderungsbehörde kontrollierten Weg. ${ }^{52}$ Doch auch auf der spanischen Seite herrschte ein durchaus ambivalentes Verhältnis zur individuellen Abwanderung, gaben die staatlichen Wirtschaftspläne doch regelrechte Planziele für die jährlich ins Ausland zu sendenden Arbeitskräfte vor, um entsprechende Devisengewinne durch Lohnüberweisungen zu realisieren. Vor diesem Hintergrund stand während der 1960er Jahre hinter der offiziellen Linie vollkommen kontrollierter Abwanderung zumindest eine Duldung der inoffiziellen Arbeitsmigration, die dazu beitrug, trotz eines schwerfälligen bürokratischen Apparates eine hohe Migrationsintensität zu erzielen. ${ }^{53}$ Der Regulierungsgrad, den die spanische Wanderungsbehörde in Bezug auf die Arbeitsmigration nach Deutschland dennoch erlangte, besaß vor diesem Hintergrund unter strategischen Gesichtspunkten für die auf die binnenwirtschaftliche Entwicklung gerichtete Zielsetzung der Migrationspolitik einen hohen Stellenwert.

Die Entwicklung in Griechenland bot nahezu zeitgleich bei ähnlichen Absichten und Ansätzen in der Realität ein gänzlich anderes Bild. Dort hatte zwar bereits 1951 eine staatliche Kommission begonnen, an der Formulierung einer künftigen Migrationspolitik zu arbeiten, und man beteiligte sich an verschiedenen internationalen Initiativen zur Unterstützung der Abwanderung aus Europa nach Übersee. ${ }^{54}$ Zugleich kalkulierte schon der erste Entwicklungsplan der Regierung für die Jahre 1950 bis 1953 mit der Förderung temporärer Arbeitsmigration als Teil der Wiederaufbaustrategie. ${ }^{55}$ Es gelang Griechenland jedoch zu keinem Zeitpunkt, eine erfolgreiche Kontrolle des Abwanderungsprozesses hinsichtlich der eigenen binnenwirtschaftlichen Interessen zu etablieren. Insbesondere eine Abstimmung der Anwerbung mit der Struktur des im Inland verfügbaren Arbeitskräftepotentials unterblieb. ${ }^{56}$ Regierung, Arbeitgeber, Gewerkschaften und Parteien kämpften um die Umsetzung ihrer Partikularinteressen, während sich keine Institution als stark genug erwies, eine kohärente Politik umzusetzen. ${ }^{57}$ So unterstützte die griechische Arbeitsverwaltung die Rekrutierungskampagnen ausländischer Stellen, agierte dabei jedoch eher als ein Dienstleister. Auf diese Weise konnte weder eine regionale noch eine berufsspezifische oder arbeitsmarktpolitische Beeinflussung der Abwanderung von Seiten Griechenlands gelingen. Die Zusammensetzung des Abwandererstroms determinierten daher vor allem individuelle Migrationsentscheidungen und die Selektionskriterien bzw. die Nachfrage der anwerbenden Staaten, die sich aus dem griechischen Bewerberpotential bedienen

\footnotetext{
${ }^{50}$ Ewenczky, Espagnoles, S.82f.

${ }^{51}$ Sanz Díaz, „Illegale“, S.79f.

52 Bernitt, Rückwanderung, S.12.

${ }^{53}$ Sanz Díaz, „Illegale“, S.29f.

${ }^{54}$ Lina Venturas, Greek Governments, Political Parties and Emigrants in Western Europe, in: Revue européenne des migrations internationales 17 (2001), S.43-65, hier S.46.

55 Glytsos/Katseli, Greek Migration, S.354.

56 Alexiou, L'immigration greque, S.279f.

${ }^{57}$ Ioanna Laliotou, Greek Diaspora, in: Melvin Ember u.a. (Hrsg.), Encyclopedia of Diasporas, Bd.1, New York 2005, S.85-92.
} 
konnten. Dieser hohe Freiheitsgrad im Hinblick auf die Migrationsentscheidung im Verbund mit der Selektionsmacht der Anwerbestaaten trug dazu bei, dass zwar eine Mehrheit der Migranten aus den unterentwickelten ländlichen Gebieten stammte, sich aber auch Facharbeitskräfte und Spezialisten motiviert durch die im Ausland besseren Verdienstmöglichkeiten zur Abwanderung entschieden, die zuvor in Griechenland nicht arbeitslos gewesen waren. Wenige Jahre nach Beginn der Anwerbung durch Deutschland resultierte daraus stellenweise bereits eine Verknappung von Arbeitskräften. ${ }^{58}$ Zwar schränkte Griechenland 1964 den Personenkreis ein, den es zur namentlichen Anforderung zuließ, ähnlich wie im Falle Spaniens konterkarierte die großzügige Zulassungspraxis Deutschlands jedoch diesen Versuch, den eigenen Einfluss auf die Wanderungsbewegung auszuweiten. ${ }^{59}$ Bis zum Anwerbestopp 1973 blieben die Zuständigkeiten im migrationspolitischen Handlungsfeld zersplittert, in dem die griechischen Behörden eher hilflos auf Veränderungen der Anwerbepolitik reagierten als durch koordiniertes Handeln der beteiligten Akteure nachhaltigen Einfluss zu gewinnen. ${ }^{60}$

Nun ließe sich argumentieren, dass die Abwesenheit von Lenkung grundsätzlich gut sei, da sie Migrationsentscheidungen dem Individuum bzw. einem liberalen Markt überlässt. Angesichts der Vielzahl institutioneller Akteure, die Einfluss auf jeden, insbesondere aber jeden regulierten Wanderungsprozess nehmen, wäre dem entgegenzuhalten, dass die Abwesenheit von Einflussnahme auf der einen Seite nicht notwendigerweise dem Individuum größere Freizügigkeit, sondern der Steuerung von Wanderung durch die jeweils andere Partei Spielraum verschafft. Die Bedeutung staatlicher Strukturen, die geleitet von den Maßgaben internationaler Standards die Parameter einer Wanderungsbeziehung aushandeln und überwachen - hierbei besteht freilich keine automatische Übereinstimmung zwischen den Interessen eines Staates und seiner abwanderungswilligen Bürger erweist sich so als vielschichtig. Einerseits kann der Staat bei stark abweichenden Interessenlagen zum Antagonisten von Ab- oder Zuwanderern werden, andererseits kann er sich - bei einer gewissen Kongruenz der Interessen - als ihr wichtigster Anwalt und Fürsprecher erweisen. Im Falle Griechenlands bildete sich letztlich ein großer Bewerberpool, mit dem die deutsche Anwerbebürokratie nach ihrem Gutdünken verfahren konnte. So flossen Griechenland bald deutsche Vermittlungsaufträge zu, die in Italien und Spanien nicht erfüllbar waren. Die deutschen Stellen hofften, sie in Griechenland aufgrund des noch unabgeschöpften Arbeitskräftepotentials, der geringen Regulierungsintensität auf griechischer Seite und dem Fehlen jeglicher ernsthafter Konkurrenz erledigen zu können. Dabei lag die Ablehnungsquote der deutschen Anwerbekommission zeitweise über 40 Prozent. Die griechischen Migranten hatten also im Selektionsprozess keine gute Position und insbesondere keine Rückendeckung durch ein Verfahren, das etwa der griechischen Seite Mitsprache bei der Beurteilung von gesundheitlicher Tauglichkeit und beruflichen Qualifikationen einräumte.

Vergleichend deuten die Statistiken der Bundesanstalt für Arbeit auf eine quantitativ nahezu gleichbedeutende Zuwanderung aus Spanien und Griechenland hin, bei der sich auch der Anteil der direkt angeworbenen Arbeitskräfte, also der dem Selektionsprozess am weitesten unterworfenen Migranten, mit rund 69 Prozent im Falle Spaniens bzw. 65 Prozent der griechischen Arbeitswanderer, ebenfalls kaum unterschied. Für beide Staaten ergibt sich mithin ein ähnlich hoher Anteil regulierter Migration, im Falle Spaniens lag die Ver-

\footnotetext{
${ }^{58}$ Ayse Kudat/Marios Nikolinakos, A Comparative Study in Migration Policies. The Case of Turkey and Greece, Berlin 1975, S.31f.

${ }^{59}$ Xenophon Zolotas, International Labor Migration and Economic Development. With special Reference to Greece, Athen 1966, S.52f.

${ }^{60}$ Venturas, Greek Governments, S. 47.
} 
teilungsmacht jedoch in hohem Maß beim Entsendestaat, während in Griechenland der Anwerbestaat Deutschland den Mobilisierungs- und Selektionsprozess weitgehend nach seinen Interessen gestalten konnte.

\section{Erkenntnisse}

Die Bundesrepublik eröffnete mit den beiden Abkommen im März 1960 die erste Runde der Expansion ihrer formalisierten Migrationsbeziehungen. Sie verschaffte sich Zugriff auf zwei Arbeitsmärkte mit bedeutendem Abwanderungspotential in einer Situation, in der die Anwerbung in Italien bereits an Bedeutung zu verlieren begann, der Bedarf der bundesdeutschen Wirtschaft an zusätzlichen Arbeitskräften jedoch noch seinem Höhepunkt zustrebte. Spanien wurde ein nicht unwichtiger Wanderungspartner für Westdeutschland, seine Bedeutung lag in der Rückschau jedoch eher im Überbrücken der Lücke, die bis zum Beginn der Anwerbung in der Türkei und in Jugoslawien auf dem deutschen Arbeitsmarkt entstand. Dabei unterwarf sich die Bundesanstalt für Arbeit in Spanien einem stark von dortigen Stellen beeinflussten Mobilisierungs- und Selektionsprozess. Auf der anderen Seite besaß die Abwanderung nach Deutschland für Spanien nicht nur eine quantitative, sondern aufgrund des hohen Steuerungspotentials auch eine große strategische Bedeutung. Ein Indiz hierfür ist die ab 1965 bereits wieder rückläufige Präsenz spanischer Arbeitnehmerinnen und Arbeitnehmer in Deutschland, während zugleich die Abwanderung aus Spanien insgesamt noch nicht ihren Höhepunkt erreicht hatte. Sie diversifizierte sich vielmehr nicht nur in der zweiten Hälfte der 1960er Jahre, sondern verlagerte sich auch wieder stärker in Richtung des traditionell wichtigsten Migrationspartners Frankreich.

Griechenland nahm für den deutschen Arbeitsmarkt im Grunde zunächst eine vergleichbare Position ein. Die Anwerbung in Griechenland erfolgte in den 1960er Jahren indes nahezu unter den Bedingungen eines deutschen Monopols. Insofern spielte die Arbeitsmigration aus Griechenland für die deutschen Bedarfsdeckungsstrategien durch die Orchestrierung seiner sich ausweitenden Migrationsbeziehungen eine besondere Rolle, da eine fehlende Interessenvertretung auf griechischer Seite das dortige Arbeitskräftepotential zu einer willkommenen Dispositionsmasse machte. Griechenland geriet auf diese Weise geradezu in Abhängigkeit von Deutschland und konnte den Wanderungsprozess kaum in seinem Sinne oder im Interesse seiner Auswanderer beeinflussen. Auch hier sprechen nicht zuletzt die statistischen Befunde für diese Interpretation, zeigen sie doch, dass die Zuwanderung aus Griechenland ihren Netto-Höhepunkt erst 1970 erreichte.

Jenseits solcher Befunde, die sich unmittelbar aus der Analyse von Fallstudien ableiten lassen, bleiben vier Erkenntnisse auf einer übergeordneten Ebene festzuhalten. Erstens erweist es sich als produktiv, neben länderspezifischen Fallstudien Ansätze zu verfolgen, die stärker komparativ oder systembezogen vorgehen, um einige noch zu wenig erforschte Aspekte der europäischen Arbeitsmigration des 20. Jahrhunderts in den Blick zu nehmen. Das gilt etwa für das Auftreten von Konkurrenzeffekten innerhalb des komplexen Geflechts bilateraler Abkommen, das zwischen 1919 und 1974 einen internationalen Arbeitsmarkt in Europa rahmte, auf dem es letztlich keine rein bilateral bestimmten Migrationsverhältnisse geben konnte. Dies offenbart, zweitens, die große und in der Migrationsforschung bisweilen unterschätzte Bedeutung von Institutionen. Nicht nur die Rekrutierungsabkommen bzw. Wanderungsverträge verdienen neue Aufmerksamkeit in einem internationalen Kontext, auch Aus- bzw. Einwanderungsbürokratien und Migrationsregime gilt es, im europäischen Kontext intensiv zu untersuchen - ohne dem Kurzschluss zu erliegen, die autoritären Staaten im Südwesten Europas seien mit ihren Strukturen als ein Erfolgs- 
modell ökonomisch sinnvoll oder effizient gesteuerter Abwanderung zu verstehen. So ist, drittens, die Regulierung von Arbeitsmigration durch ein System bilateraler Anwerbeabkommen keineswegs uneingeschränkt als gerecht, human oder ökonomisch sinnvoll zu bezeichnen. Als Vorstufe von freizügigen multilateralen Arbeitsmärkten einerseits und des Übergangs Europas zum Einwanderungskontinent andererseits haben sie sich aber in ihrer Zeit in gewisser Hinsicht als für alle Beteiligen - auch die Migranten - durchaus vorteilhaft erwiesen und erfreuen sich bis heute einer weltweit steigenden Bedeutung. ${ }^{61}$ Als problematisch hat sich, auch in unseren Fallbeispielen, weniger die Mobilisierung und der Transfer von Arbeitskraft durch diese Institution erwiesen als vielmehr ihre Mittäterschaft an der Suggestion, es könne temporäre Zuwanderung ohne partielle Einwanderung geben, und dem daraus resultierenden Versäumnis vieler beteiligter Staaten, sich der schnell offensichtlich werdenden Einwanderungssituation zu stellen. Viertens zeigen die Befunde zu Spanien und Griechenland, dass es weiterführend sein könnte, im Hinblick auf die Geschichte der innereuropäischen Arbeitsmigration den Abwanderungsländern bei der Prozessgestaltung größere Bedeutung zuzumessen und dabei nicht nur die Art und Weise $\mathrm{zu}$ beobachten, in der diese mit dem Phänomen umgingen, sondern auch die dem zugrunde liegenden Kausalitäten. Das erfordert die Rückkehr von der Systemebene zu den politischen, sozialen, ökonomischen und gesellschaftlichen Prozessen, die in jedem Staat hinter den in diesem Beitrag stark induktiv beschriebenen Zusammenhängen standen, und umreißt eine der Herausforderungen der Historischen Migrationsforschung in Europa.

${ }^{61}$ Rass, Bilaterale Wanderungsverträge, S. 100f. 
